\title{
3

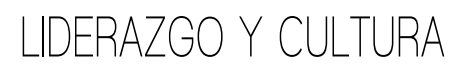 ORGANIZACIONAL
}

María Constanza Aguilar Bustamante*

Universidad Santo Tomás

El liderazgo y la cultura organizacional son temas que despiertan un gran interés en el mundo de las empresas. La relación que se aborda en este trabajo es desde la perspectiva de algunos autores: son los líderes los que determinan y conforman la cultura organizacional (Bass, 1985; 1990; 1999; Contreras, Barbosa y Piñeros, 2016; Contreras, Barbosa, Juárez y Uribe, 2010; Moss, 2010; Ogliastri, 1999). Lo que deja en evidencia la literatura científica, por ejemplo, en los estudios transculturales e internacionales, es una importante relación entre la cultura organizacional y los estilos de liderazgo (Anastácio Barata y Martí Ripoll, 2015; Contreras, Barbosa y Piñeros, 2016; Gil y Martí, 2011; Hanges y Dickson, 2004; Dorfman y Hanges, 2004). A continuación, se presenta una síntesis sobre el recorrido histórico de ambos temas, sus desarrollos y avances.

\section{Liderazgo y líderes}

Autores como Bligh y Meindl (2005) y Glynn y DeJordy (2010) han llevado a cabo estudios sobre los líderes y el liderazgo, las variables asociadas a él; modelos teóricos y conceptuales, métodos y técnicas que se encuentran en función de las propuestas teóricas existentes, y ponen en evidencian que su desarrollo teórico aún está en proceso, que la mayor cantidad de investigación se concentra en Estados Unidos, y que frente a los conceptos de liderazgo y los líderes existe una interminable lista de términos referidos a ellos, y cuando se revisan sus definiciones siguen sin estar suficientemente claros.

Revisar la literatura científica sobre el liderazgo lleva a investigar etapas y enfoques por los que ha transitado y las dimensiones y temas que incluye; Francisco Gil (2008) afirma que

mariaaguilar@usantotomas.edu.co

* https://orcid.org/0000-0003-2990-9667 
el entusiasmo, específicamente de los académicos por este tema tiene "un movimiento pendular que va de momentos de entusiasmo a grandes desilusiones" (p. 295).

\section{Desarrollo histórico del liderazgo}

Autores como Bass (1990), Yukl (2002), Gil y García Sáiz (1993), Gil et al. (2011), entre otros, definen el concepto de liderazgo como un proceso grupal, un rasgo de personalidad, una forma de inducir al compromiso, llevar a cabo un ejercicio de influencia, comportamientos y estrategias para persuadir a otros, presentar una relación de poder, un instrumento para alcanzar metas, un efecto de la continua interacción, tener un rol diferenciado, un rol de poder asignado (como cuando se es jefe y se tienen personas a cargo), y todas las posibles combinaciones de las anteriores.

A continuación, se presenta un breve recorrido cronológico, y algunos conceptos que se encuentran asociados al liderazgo.

De acuerdo con Peiró (1995), los inicios de la investigación empírica en liderazgo aparecieron con Kurt Lewin en 1940, cuando él y algunos de sus discípulos trabajaban en el tema. En esta primera etapa prevalecieron los estudios del enfoque de rasgos, en los que el tema central se refiere a que los líderes nacen, y el liderazgo es una habilidad innata (Bryman, 1996).

Luego, en los años cincuenta, comienzan a asociarse una gran cantidad de conceptos al liderazgo, específicamente sobre los estilos de liderazgo, algunos de ellos son: liderazgo funcional, liderazgo sin líder, liderazgo burocrático, carismático, democrático, autocrático, laissez, entre muchos otros; Peiró (1995) pone en evidencia que a pesar de la variedad no hay consenso sobre su definición ni forma de estudiarlo. Según Bryman (1996), la característica principal de estos estudios puede resumirse en la pregunta que muchos trabajos intentaron responder sobre qué hacen y cómo son los líderes.

En la década de los setenta y principios de los ochenta, sobresale la obra de Stogdill (1974) quien publicó un handbook sobre el liderazgo. Este autor llevó a cabo una revisión durante cuatro décadas, sobre "todo" lo que se había publicado alrededor del liderazgo, mostrando, luego igual que Peiró (1995), unas décadas más tarde, que aún no se cuenta con una comprensión integrada al liderazgo.

En estos años surge el enfoque de contingencia, con un concepto nuevo: la eficacia del liderazgo está afectada y determinada por el contexto y la situación (Bryman, 1996). Al detallar la cronología durante las décadas de los ochenta y noventa, según Glynn y Dejordy (2010), nacen dos propuestas teóricas: 1) las teorías comportamentales y 2) las teorías contingenciales, que son las que dominan las publicaciones; después de estas décadas no existen perspectivas teóricas dominantes. Sin embargo, después de los años 
ochenta surgen trabajos sobre un nuevo liderazgo: el liderazgo transformacional y carismático, es decir que los líderes deben desarrollar una visión estratégica, han de inspirar lealtad en sus seguidores y, además, se deben implicar emocionalmente (Bryman, 1996).

En los noventa y siguientes se desarrollan tendencias actuales sobre el liderazgo y sus perspectivas asociadas al estudio de otros procesos psicológicos a nivel individual, grupal y organizacional. Algunas de ellas son bienestar, calidad de vida, comunicación, grupos y equipos de trabajo, los aspectos éticos del liderazgo, liderazgo por e-learning, liderazgo como fenómeno grupal, liderazgo como proceso, líder como sujeto, los seguidores, la situación, entre otras; incluyendo el factor cultural como uno de sus elementos fundamentales, teniendo en cuenta los procesos de globalización que se dan en el mundo (Contreras, Barbosa y Piñeros, 2016; Molero y Morales, 2011; Sabucedo y Morales, 2015).

Por otro lado, Aguilar y Correa (2017); Antonakis, Cianciolo y Stemberg (2004); Contreras (2008); García, Aguilar, Payan y Forero (2016); Molero (2011); Gil y Alcover (2008); Sabucedo y Morales (2015), demostraron, luego de llevar a cabo análisis bibliométricos y revisiones sistemáticas, una gran diversidad de formas de investigar en el liderazgo en el marco de la psicología social y de las organizaciones; estos autores muestran que la investigación sobre el liderazgo está en fase de desarrollo y se encuentra una gran heterogeneidad en estudios cuantitativos, cualitativos y esto puede dificultar la manera como se presentan y dan a conocer los resultados de las investigaciones por parte de las comunidades académicas.

Los estudios de liderazgo que utilizan metodologías cuantitativas usan principalmente cuestionarios, por ejemplo, Bass y su equipo, han estudiado el liderazgo transformacional (Bass, 1985; 1990; 1999; Bass y Avolio, 1994). La mayoría de los cuestionarios son de dos tipos: a) dirigido a los líderes (y se hace de forma autoadministrada), y b) dirigido a los empleados o colaboradores (quienes evalúan las características de su líder). Los ítems corresponden a un modelo teórico que respalda y hace énfasis en determinadas dimensiones, características o variables que permiten medir el liderazgo. Más adelante se presentan los desarrollos históricos del liderazgo.

La investigación del liderazgo a través de experimentos no es muy usual, dada la rigurosidad del diseño de la situación controlada y la manipulación de las variables que el investigador debe tener. Un ejemplo de esto en un contexto real es Lewin, Litpit y White (1939), citados por Peiró (1995), quienes llevaron a cabo un experimento de campo, utilizando muestras de estudiantes. Esto, hoy por hoy, es muy difícil de replicar teniendo en cuenta las exigencias éticas.

Los estudios cualitativos en el liderazgo han usado estrategias como observación participante y entrevistas en profundidad que, de acuerdo con Schilling (2007), es la que más se usa. 
Molero y Morales (2011) y Molero (2011) muestran que los estudios cuantitativos y cualitativos que se conocen sobre el liderazgo y se llevan a cabo desde una perspectiva científica, intentan estudiar el comportamiento del líder, los seguidores y la situación. Cada metodología tiene puntos a favor y en contra, y como el liderazgo es tan complejo, necesita que se integren y complementen; la rigurosidad de ambas metodologías permite replicar y validar investigaciones para contrastar la información que se encuentra en la evidencia publicada.

Ahora bien, pero, ¿qué es el liderazgo? Se presentan dos definiciones, de las muchas encontradas en la literatura, pero estas dos son de autores que han sido importantes en el desarrollo del estudio y las propuestas teóricas del liderazgo, Bass (1990) y Yukl (2002). Bass (1990) define el liderazgo:

[...] como un proceso de interacción entre dos o más personas que normalmente implica estructurar o reestructurar la situación, percepciones y expectativas; el líder es un agente de cambio (influye más en las conductas de otros, que estos sobre él), y el liderazgo tiene lugar cuando un miembro del grupo modifica la motivación o competencias de los miembros del grupo. (pp. 20-21)

Yukl (2002) define el liderazgo como:

[...] el proceso de influir en otros para que entiendan y estén de acuerdo acerca de las necesidades que se deben atender y cómo hacerlo de forma efectiva, y el proceso de facilitar esfuerzos individuales y colectivos para alcanzar objetivos compartidos. (p. 7)

No se olvide que cuando se estudia el liderazgo son tres los elementos que se deben identificar: el líder, los seguidores y el contexto (Aguilar y Correa, 2017). Para finalizar este apartado se presentan cuatro clasificaciones sobre los desarrollos y las aproximaciones teóricas al estudio del liderazgo de acuerdo con Gil (2008):

- Las teorías clásicas de rasgos, estilos de liderazgo y contingencias/situacionales;

- los enfoques alterativos, con una gran influencia el modelo médico;

- los enfoques recientes, con sólidas bases en lo estratégico, carismático, transformacional, transaccional; $y$

- las orientaciones actuales en la dirección y el liderazgo.

Aguilar (2017) y Gil (2008) presentan un resumen de estas clasificaciones, se sintetizan sus autores, principios, aportes y críticas al desarrollo del liderazgo. 
Tabla 1. Teorías clásicas: rasgos, estilos y contingencia

\begin{tabular}{|c|c|}
\hline \multirow{2}{*}{$\begin{array}{l}\text { El liderazgo } \\
\text { como rasgo de } \\
\text { personalidad }\end{array}$} & $\begin{array}{l}\text { - Rasgos personales estables que definen a los líderes. } \\
\text { - Carácter innato de ese rasgo. } \\
\text { - Los líderes nacen. }\end{array}$ \\
\hline & $\begin{array}{l}\text { - Críticas (Yukl y Van Fleet, 1992) no se demostró que un conjunto de rasgos relevantes sea } \\
\text { universal. } \\
\text { - Relación entre rasgo y éxito es escasa e inconsistente. }\end{array}$ \\
\hline \multirow[t]{2}{*}{$\begin{array}{l}\text { El liderazgo } \\
\text { como conducta }\end{array}$} & $\begin{array}{l}\text { - Importante cómo se comporta el líder. } \\
\text { - Cuáles son sus conductas y estilos de comportamiento. } \\
\text { - Las conductas son responsables de la eficacia del liderazgo y pueden aprenderse. } \\
\text { - Dos grandes programas de investigación: } \\
\text { - Estudios de la Universidad de Ohio (Hemphill, Flleishman, Stodgill, Shartle y Pepinsky, } 1962 \\
\text { citados por Peiró, 1995), identificar dimensiones para caracterizar los principales factores en } \\
\text { la conducta de los líderes. } \\
\text { - Estudios de la Universidad de Míchigan (Kahn y Katz, 1978), líderes centrados en los } \\
\text { empleados y líderes centrados en la producción. }\end{array}$ \\
\hline & $\begin{array}{l}\text { - Críticas (Bryman, 1996) a este enfoque orientadas a señalar las dificultades en las medidas, } \\
\text { problemas para identificar la causalidad (estilo de liderazgo se relaciona con rendimientos de } \\
\text { empleados), ausencia de variables situacionales, entre otros. }\end{array}$ \\
\hline \multirow[t]{2}{*}{$\begin{array}{l}\text { Enfoques } \\
\text { contingentes: la } \\
\text { influencia de las } \\
\text { situaciones }\end{array}$} & $\begin{array}{l}\text { - Se denominan modelos contingentes o situacionales. } \\
\text { - La eficacia del liderazgo depende de las condiciones de la situación en que sucede. } \\
\text { - Teoría de la contingencia (Fieldler, 1967), relación líder-miembros, posición de poder del } \\
\text { líder, estructura de la tarea. } \\
\text { - Teoría de liderazgo situacional (Hersey y Blanchard, } 1969 \text { citados por Peiró, 1995), el modelo } \\
\text { predice una evolución en los estilos de liderazgo, desde decir hasta delegar, y parte de la } \\
\text { premisa del desarrollo o madurez de los subordinados. } \\
\text { - Teoría camino-meta (House, 1996, citado por Peiró, 1995), la eficacia del liderazgo } \\
\text { depende de tres variables, las conductas de los líderes (cuatro tipos de conducta: centrada } \\
\text { en la tarea, de apoyo, participativa y orientada al logro), los rasgos de los subordinados } \\
\text { (dependencia, autoritarismos, habilidad, locus de control) y determinadas variables } \\
\text { situacionales (estructura de la tarea, ambigüedad del rol, autonomía y ámbito del trabajo e } \\
\text { interdependencia de la tarea). } \\
\text { - Modelo de liderazgo participativo (Vroom y Yetton, 1973), se relaciona la conducta de } \\
\text { liderazgo y la participación para la toma de decisiones; también se le conoce como modelo } \\
\text { normativo (Vroom y Jago, 1978), analiza el estilo de decisión de los líderes (autocrático, } \\
\text { consultivo, grupal y delegativo) en función de situaciones. } \\
\text { - Teoría de los recursos cognitivos (Fiedler y García, 1987), la eficacia del liderazgo depende } \\
\text { de los recursos cognitivos del líder (inteligencia y experiencia) y las demandas de las } \\
\text { situaciones (complejidad de la tarea). } \\
\text { - Teoría de los sustitutos de liderazgo (Kerr y Jernier, 1978). }\end{array}$ \\
\hline & $\begin{array}{l}\text { - Críticas a los enfoques contingentes dado que han surgido listas interminables de factores } \\
\text { situacionales. Obtienen evidencia parcial o indirecta (Gil y Alcover, 2008). }\end{array}$ \\
\hline
\end{tabular}

Fuente: Aguilar (2017) 
La segunda clasificación sobre las teorías del liderazgo, Aguilar (2017) y Gil (2008), corresponde a los enfoques alternativos.

Tabla 2. Enfoques alternativos del liderazgo

$\begin{array}{cl}\text { Reanimar } & \text { - Frente a los problemas que suscitaron los modelos contingenciales, autores como Smith y } \\ \text { la teoría del } & \text { Peterson (1988, p. 47), con base en el modelo médico, proponen aplicar ciertos "remedios" } \\ \text { liderazgo } & \text { para reanimar la teoría del liderazgo. } \\ & \text { 1. Reemplazar las medidas del estilo del líder por medidas de recompensa y castigo. } \\ & \text { 2. Hacer distinciones entre subordinados (teoría del intercambio líder-seguidores, LMx), } \\ & \text { concepto de endogrupo y exogrupo (Graen y Uhl-Bien, 1995). } \\ & \text { 3. Reexaminar las bases de las percepciones de los subordinados respecto a los líderes } \\ & \text { (importantes las atribuciones que realizan los líderes). } \\ \text { 4. Reexaminar las bases de las percepciones de los subordinados respecto a los líderes (las } \\ \text { personas tienen sus propias creencias - teorías implícitas-. } \\ \text { 5. Revisar las circunstancias que determinan el liderazgo. } \\ \text { 6. Podemos señalar una propuesta más y es la hecha por Den Hartog y Koopman (2001), } \\ \text { quienes analizan el liderazgo a partir de las tácticas de poder e influencia. La aplicación } \\ \text { de los recursos de poder resulta de gran importancia para explicar el comportamiento de } \\ \text { los líderes (Yukl y Van Fleet, 1992). }\end{array}$

Fuente: Aguilar (2017)

En este segundo momento, de acuerdo con Smith y Peterson (1988), hay una tendencia a estudiar el liderazgo y retomar elementos de la psicología social, psicología cognitiva, psicología organizacional y de otras ciencias sociales; se le da mayor importancia a la forma como el líder y los seguidores perciben e interpretan las acciones del otro, y como esto genera un proceso de influencia; y, además, utilizan metodologías mixtas, experimentos de laboratorio, de campos y estadísticas cada vez más sofisticadas.

La tercera clasificación presenta tres temas importantes: el liderazgo estratégico, nuevamente los estudios sobre los rasgos y los nuevos liderazgos (Aguilar, 2017; Gil, 2008).

Tabla 3. Enfoques recientes de liderazgo

\section{Se caracteriza por:}

Liderazgo estratégico
- Preocupación por los líderes y directivos de niveles medio y superior.

- Dirigido a equipos de la alta dirección encargados de afrontar los nuevos retos de la globalización.

- Respuestas organizacionales frente a las condiciones del ambiente y se relaciona con un liderazgo para toda la empresa.

- Los líderes estratégicos ejercen una gran influencia, exagerada atribución a su poder; importante la cultura organizacional, los recursos disponibles, equipos ejecutivos, buscan consenso de grupo, una sola persona no puede hacer frente a todo, de ahí la necesidad de fortalecer los grupos (Yukl, 2002; Aditya, House y Kerr, 2000; Gil, 2008). 
Reaparece el interés por el estudio de los rasgos años ochenta.

Revitalización de - A través de metaanálisis se confirma la importancia de rasgos, inteligencia, masculinidad, los estudios de dominancia, en el aumento del éxito del líder, aunque no lo garanticen (Aditya, House y rasgos Kerr, 2000; Yukl y Van Fleet, 2002).

- Surgen teorías que relacionan el liderazgo con las situaciones: la teoría de Mischel (1973) citado por Peiró (1995), distingue situaciones débiles y fuertes, en las que el líder debe mostrar sus tendencias disposicionales. La teoría de motivación de poder (McClelland, 1975, citado por Peiró, 1995) que es un antecedente del liderazgo carismático, y permite identificar un perfil de liderazgo eficaz, con determinados rasgos, como un alto nivel de motivación de poder (afiliación) con una alta preocupación por el uso moral del poder.

Nuevo liderazgo - Luego de los años ochenta surge un renovado interés por el liderazgo y esto da lugar a nuevas teorías.

- Surgen el liderazgo carismático y el liderazgo transformacional.

- El liderazgo carismático desarrollado por Weber (1929) y retomado luego por House y Aditya (1997) describe una forma de influencia basada en las percepciones que los seguidores tienen del líder y que se produce en momentos de crisis social.

- La teoría del autoconcepto (House y Aditya, 1997; Shamir, House y Arthur, 1993) explica el liderazgo carismático basándose en un conjunto de rasgos y conductas identificadas en los líderes que influyen en las actitudes y conductas de los seguidores.

- Se reconoce el "lado oscuro del liderazgo carismático", con numerosos ejemplos de líderes políticos y de sectas milenaristas (Gil, 2008).

- Se identifican otros tipos de carisma (Howell y House, 1992, citado por Aditya, House y Kerr, 2000): personalizado, el cual se caracteriza por ser autoagradecido, explotador y autoritario. Carisma socializado, es altruista, colectivista e igualitario (Bass, 1999).

- El desarrollo del liderazgo transformacional, que proviene de la diferencia entre liderazgo transaccional y transformador.

- El liderazgo transaccional implica un contrato implícito entre líderes y seguidores que establece un intercambio (el líder otorga recompensas, dinero, prestigio, etc.). El liderazgo transformacional eleva las aspiraciones de sus seguidores, las funde con las suyas propias y les estimula y motiva (Bass y Avolio, 1994; Gil, 2008).

- Bass $(1985 ; 1999)$ elabora la teoría de liderazgo transformacional a partir de la distinción transaccional-transformador. Esta propuesta resulta efectiva en todas las situaciones y culturas e incluye dimensiones como: carisma, motivación inspiracional, consideración individualizada y estimulación intelectual (Gil, 2008).

- Yukl (2002) cuestiona que este estilo de liderazgo sea una panacea que pueda resolver todos los problemas de las grandes organizaciones, la mayoría de las investigaciones han evidenciado que los cambios en las organizaciones no se deben tanto a los líderes carismáticos, como a los procesos transformacionales de liderazgo.

Fuente: Aguilar (2017)

La cuarta perspectiva (Aguilar, 2017; Gil, 2008) se refiere a temas actuales, que incluyen dirección y liderazgo, globalización y generación de nuevas tecnologías de la información, dado que han provocado grandes transformaciones en las organizaciones, y han hecho que se reestructuren funciones, áreas, roles y competencias en las empresas. Shamir y Howell (1999) consolida nuevos escenarios, alternativos de liderazgo: 1) liderazgo indispensable, de usar y tirar; corresponde a un acuerdo temporal, limitado en su objetivo y duración. 2) Liderazgo colectivo, compartido, distribuido o 
de los miembros (importante, ya que plantea que el liderazgo se distribuye entre los miembros que lo ejecutan, es decir, equipos autónomos). 3) El novedoso teleliderazgo, en el que el líder debe centrarse en desarrollar una comunicación más efectiva; se relaciona con la transmisión de información entre el líder y seguidores a través de las tecnologías de comunicación mediada y los sistemas de apoyo a la decisión grupal.

\section{Tabla 4. Temas y orientaciones actuales}

\begin{tabular}{|c|c|}
\hline $\begin{array}{l}\text { Liderazgo } \\
\text { distribuido o } \\
\text { compartido }\end{array}$ & $\begin{array}{l}\text { - Propuesto por autores como Aditya, House y Kerr (2000) y Bryman (1996), adopta } \\
\text { diferentes modalidades y contrasta un liderazgo carismático con enfoques de nuevos } \\
\text { liderazgos - liderazgo de personas heroicas, de alto nivel y está centrado más en las } \\
\text { personas que en los grupos-. } \\
\text { - Algunos ejemplos son liderazgo delegado, coliderazgo, liderazgo de los miembros, } \\
\text { superliderazgo (Manz y Sims, 1989), modelo de los equipos reales (Katzenbach y Smith, } \\
\text { 1993), modelo de liderazgo (Kouzes y Posner, 1995; 2002), procesos y habilidades de } \\
\text { liderazgo (Hosking, 1988). Estos modelos por lo general se proponen alrededor de una } \\
\text { idea común, y es hacer que los miembros del grupo sean capaces de liderarse a sí mismos } \\
\text { (Manz y Sims, 1989). }\end{array}$ \\
\hline $\begin{array}{l}\text { Gestión de la } \\
\text { diversidad }\end{array}$ & $\begin{array}{l}\text { - Esta perspectiva reconoce diversas fuerzas de trabajo y miembros en cuanto a raza, etnia, } \\
\text { edad, género, formación, nivel socioeconómico, entre otros, lo que implica importantes } \\
\text { beneficios, pero también, otros costos y muchos conflictos (Alcover, 2008). } \\
\text { - Los líderes tienen el reto de gestionar la diversidad y encontrar el balance adecuado en la } \\
\text { organización para promover una fuerte identidad y una cultura organizacional basada en } \\
\text { valores e identidad compartida (Gil, 2008). }\end{array}$ \\
\hline $\begin{array}{l}\text { Liderazgo y gestión } \\
\text { del conocimiento }\end{array}$ & $\begin{array}{l}\text { - De acuerdo con Davenport (2000) y Delgado y Castañeda (2011), la ventaja competitiva de } \\
\text { las organizaciones radica en la capacidad que tienen de aprender, así deberá gestionar su } \\
\text { capital intelectual, relacional, y desarrollar un clima organizacional que propicie compartir la } \\
\text { información en todos los niveles y áreas de la organización, y generar conocimiento. }\end{array}$ \\
\hline Liderazgo ético & $\begin{array}{l}\text { - Yukl (2002) se acerca a proponer unos criterios para evaluar el liderazgo ético, dado } \\
\text { que resulta importante conocer los esfuerzos que hacen los líderes para mostrar un } \\
\text { comportamiento ético y para erradicar las prácticas no éticas en las organizaciones. } \\
\text { Evaluarlo es complejo y requiere identificar valores, intenciones y la moral de los líderes } \\
\text { (Gil, 2008). }\end{array}$ \\
\hline $\begin{array}{c}\text { Liderazgo en } \\
\text { distintas culturas }\end{array}$ & $\begin{array}{l}\text { - Gil (2008) afirma que el significado que se les da a los líderes varía de acuerdo con el } \\
\text { contexto cultural, y que la mayoría de las teorías sobre el liderazgo surgen en Estados } \\
\text { Unidos, y los estudios reflejan el interés por valores individualistas, posturas alrededor de } \\
\text { la racionalidad, incentivos individuales, responsabilidades de los seguidores, motivación } \\
\text { hedonista, orientación a valores democráticos. } \\
\text { - Prepararse para los años siguientes, la globalización hace necesario que los líderes } \\
\text { entiendan los valores de cada cultura. } \\
\text { - Aquí surge el proyecto más extenso y ambicioso que estudia el liderazgo en diferentes } \\
\text { culturas, el proyecto Globe que se presenta más adelante (Gil Rico, 2000). }\end{array}$ \\
\hline
\end{tabular}

Fuente: Aguilar (2017) 


\section{Cultura}

Para la psicología del trabajo y las organizaciones y la psicología social, la cultura corresponde a uno de los factores más importantes de influencia sobre el comportamiento a través del intercambio de normas y valores por parte de un gran número de personas (Shiraev y Levy, 2010). Para este caso en particular, el interés de este trabajo es sobre el papel de la cultura en el liderazgo y del liderazgo en la cultura. La cultura, tal como la definen House, Wright y Aditya (1997), corresponde a una serie de procesos comunes compartidos, formas de pensar, sentir, reaccionar, significados e identidades, ambientes socialmente construidos y compartidos. De acuerdo con Contreras, Barbosa y Piñeros (2016), el líder es un elemento importante en este proceso pues depende de lo que las personas consideren es su rol ideal. El líder desempeña un papel determinante en el desarrollo de la cultura y la forma como las personas se comportan y transmiten dichos patrones de generación en generación.

Nader y Solano (2009) llevan a cabo una interesante revisión sobre los antecedentes y la relación entre liderazgo y cultura, en la que muestran que los primeros trabajos se encuentran reseñados en algunos libros de autores como Sackmann (1991), Schein (1992) y Deal y Kennedy (1982), desde los años ochenta, quienes mostraron relaciones entre la cultura organizacional, los valores y el liderazgo.

En la literatura científica se identifican dos corrientes o dos perspectivas, unos que apoyan y confirman esta relación y otros que consideran que las habilidades de los líderes no se ven influenciadas por la cultura. Nader y Solano (2009) muestran algunos autores (Adler, Doktor y Redding, 1986; Child y Tayeb, 1983; Levitt, 1983) que afirman que: "las prácticas culturales y el contexto organizacional no afectan las habilidades del liderazgo pues los atributos del líder son en su mayoría universales (éticos) y las acciones que lo hacen efectivo, trascienden el contexto" (p. 239). La segunda perspectiva que se tiene en cuenta en el desarrollo de este trabajo, y que es de gran impacto científico hoy por hoy, confirma que un líder es efectivo si es reconocido como tal y esto está en estrecha relación con los valores, las tradiciones culturales y las ideologías de los seguidores (House, Wright y Aditya, 1997; House y Javidan, 2002).

Tal como se observó en las teorías del liderazgo, al revisar la relación entre cultura y liderazgo surge la misma problemática. Siempre que se estudie el liderazgo no se pueden dejar de lado las variables del contexto y la situación donde surge este. Triandis (2004) deja en evidencia en sus trabajos transculturales la necesidad de conocer las acciones del líder que más se ajustan a las pautas y prácticas culturales vigentes en una organización. Las teorías y modelos del liderazgo son en extremo generales y 
caracterizan las acciones del liderazgo independientemente del contexto, ya sea el organizacional o el cultural más amplio (House, Javidan y Dorfman, 2001; Yukl, 2002).

En este trabajo se aborda la cultura organizacional y no la cultura social. De acuerdo con Schein (1992) y Trice y Beyer (1993), cultura y liderazgo están muy relacionados, dado que los líderes se pueden ver influenciados por las condiciones de la cultura en que se encuentran, y a la vez, un líder puede crear, modificar, cambiar, potenciar o incluso intercambiar varias culturas.

Molero (2002) afirma que el liderazgo es capaz de crear culturas cuando, por ejemplo, una persona que tiene un proyecto o una visión, crea una organización. De este modo, el proyecto de empresa, según Trice y Beyer (1993), es la "sustancia" de la cultura organizacional, siendo los creadores y dueños una parte muy importante de la cultura de la organización por toda la influencia que ejercen en ella.

Para el segundo caso, estos autores afirman que el liderazgo cambia culturas, cuando los líderes cambian a otros a través de sus conductas, son carismáticos o transformacionales y logran influir y producir cambios en las organizaciones. También puede esperarse que el líder no cambie la cultura, sino que la fortalezca, la preserve, con miras a mantener la identidad de la organización. Es importante reconocer en las organizaciones el surgimiento de subculturas, que pueden ser las áreas o unidades de una organización. De acuerdo con Molero (2002), una importante función de un líder será hacer que los individuos de cada grupo actúen de forma armónica orientados al cumplimiento de los objetivos de la organización y esto se constituye en un elemento de cultura.

$\mathrm{Al}$ revisar algunos antecedentes, modelos y teorías sobre la cultura organizacional se identificaron los siguientes. Autores como Allaire y Firsirotu (1984) llevaron a cabo una revisión sistemática e identificaron muchas teorías y las clasificaron en ocho escuelas: funcionalista, estructural funcionalista, ecológica, histórica, estructuralista, mutual, simbólica y cognitiva. De acuerdo con Martínez-Avella (2010), si se rastrean publicaciones y trabajos de corte empírico, se distinguen dos tipos de estudios sobre cultura organizacional: primero están los trabajos que identifican variables que definen la cultura organizacional y la relacionan con resultados en el ámbito organizacional; y segundo, los estudios entre culturas que buscan relacionar los rasgos de las culturas de los países con características de las culturas organizacionales.

Para el primer tipo se encuentran diferentes modelos sobre las características o rasgos que definen la cultura organizacional. Harrison (1972) es uno de los primeros autores que se encuentra en la literatura científica; él desarrolló una tipología con variables como orientación al poder, orientación al rol, a la tarea y a las personas; y 
con estos elementos describió los rasgos que definen la cultura de una organización. Cunha y Cooper (2002), diseñaron y aplicaron un cuestionario incluyendo variables como poder, rol, tarea y personas. Luego, O'Reilly, Chatman y Caldwell (1991) consideraron que los rasgos de la cultura están definidos por perfil en innovación, toma de riesgos, minuciosidad, orientación a resultados, orientación a las personas, orientación a los equipos, agresividad y estabilidad. Con esta misma orientación se identifican los trabajos de Denison y Mishra (1995), quienes dan mucha importancia a los rasgos dentro de la cultura como, por ejemplo, el involucramiento, la consistencia, la adaptabilidad y la misión.

Ahora bien, para el segundo tipo tenemos estudios entre culturas (crosscultural), el máximo autor reconocido como pionero es Hofstede (1980). Él identifica unos rasgos para comparar culturas organizacionales entre diferentes países; esto ha servido de base en múltiples estudios sobre comportamiento organizacional. Incluye variables como individualismo, distancia de poder, masculinidad, aversión a la incertidumbre; luego incorporó orientación al futuro; a través de sus trabajos se han podido organizar las diferencias culturales en patrones generalizados, y esto ha permitido llevar a cabo una investigación comparativa que ha servido de base para muchos estudios sobre cultura organizacional.

En la literatura también se observan una gran cantidad de controversias sobre su definición y contenido. Se encuentran aportes desde la psicología, la sociología, la antropología y, más recientemente, desde las ciencias administrativas (Gil y Alcover, 2008). Denison (1996), por su parte, ha aclarado el constructo de cultura organizacional y lo diferencia con el clima organizacional, puesto que en muchos textos se utilizan indistintamente. Cultura se refiere a la forma como se comporta la gente, los valores y las presunciones arraigadas y dados por supuesto; mientras que el clima organizacional hace énfasis a los factores ambientales percibidos conscientemente y sujetos al control organizacional que se traduce en normas y pautas de comportamiento. Denison (1996) y Schneider (2000) defienden la postura de que los dos constructos son complementarios.

Adicionalmente, Trice y Beyer (1993) manifiestan que la cultura incluye dos categorías. Una que se corresponde a los sistemas de creencias compartidas e interrelacionadas que tienen una alta carga emocional y que se denominan ideologías. La segunda corresponde a las formas culturales, y estas son los artefactos observables como símbolos, lenguaje, relatos y prácticas o acciones, mediante las cuales las personas de una cultura expresan, afirman y se comunican. Aquí toma mucho valor el lenguaje y todo lo que las personas transmiten a través de él, metáforas, dichos, jergas utilizadas, el argot, entre otros. 
Para Rousseau (1997) es importante señalar la participación de antropólogos y otros investigadores organizacionales en el momento de definir la cultura, teniendo en cuenta las coincidencias que tienen al definirla. Las culturas son:

\section{conjuntos de cogniciones compartidas por los miembros de una determinada unidad social, las cuales se adquieren a través del aprendizaje social y de procesos de socializa- ción que exponen a los individuos a diversos elementos culturales, como actividades e interacciones, informaciones comunicadas y artefactos materiales, que conforman las experiencias sociales al tiempo que dotan a sus miembros de valores compartidos, marcos de comprensión comunes y sistemas de creencias y de expectativas. (p. 515)}

Esto permite concluir que las culturas son dinámicas, pues cambian de forma permanente, y ejercen una serie de consecuencias sobre las personas con las que comparten. Trice y Beyer (1993) dejan en evidencia algunas consecuencias de la cultura y a través de algunos estudios que corresponden a temas como el manejo de incertidumbre colectiva, la creación de orden social, de continuidad, identidad y compromiso colectivo, fomento de etnocentrismo, entre otras.

Hay una distinción importante cuando se revisan estudios sobre cultura organizacional. De acuerdo con Smircich (1983), la diferencia entre lo que la organización es frente a la cultura como algo que una organización tiene. La primera clasificación, cultura como la organización es, recibe el nombre de enfoque "emic o simbólico"; aquí, la cultura es una metáfora, y debe captar su estructura mediante las descripciones que hacen los colaboradores de la organización; los estudios de este tipo están centrados en los significados construidos a partir de las formas culturales como, por ejemplo, rituales, símbolos, celebraciones o disposiciones del espacio físico en cada organización (Alcover, 2008).

La segunda apuesta hace referencia a la cultura como algo que la organización tiene y corresponde a elementos particulares de cada empresa como son lineamientos, las políticas, los principios, los valores y la historia; de tal forma que un observador puede reconocer fácilmente sus costumbres, símbolos, rituales, normas, entre otros; cuando se identifican estos artefactos en la cultura, se hace referencia al enfoque "etic" (Alcover, 2008). En esta misma perspectiva para Schein (1985), la cultura corresponde a las respuestas aprendidas por los colaboradores de la organización sobre los posibles problemas colectivos; es decir, la cultura es una forma de interpretar, percibir, pensar y sentir la relación de los trabajadores con los problemas. Es una forma de comportarse frente a la cotidianidad de la organización. Así, las normas los valores, los rituales e incluso el clima son mecanismos o manifestaciones de la cultura. 
De acuerdo con Alcover (2008), estas dos clasificaciones dan lugar a dos aproximaciones epistemológicas que se han utilizado para abordar el estudio de la cultura; primero, corresponden a una perspectiva interpretativa o simbólica de carácter sociocognitivo (las organizaciones son culturas, metáfora); y segundo, la postura funcionalista, de naturaleza objetivista (las organizaciones tienen culturas, y es variable), esta denominación fue propuesta por Sypher, Applegate y Sypher (1985) citado por Alcover (2008).

Otra propuesta la hace Martín (2002), quien propone ordenar las teorías sobre la cultura organizacional y las clasifica en tres: integración, diferenciación y fragmentación. La primera, integración, hace alusión a las manifestaciones consistentes y consensuadas por los miembros de la organización; y de esta manera, la cultura es aquello que resulta claro y unificado para todos los miembros. En segunda instancia, la diferenciación se refiere a las manifestaciones e interpretaciones inconsistentes; por ejemplo, en organizaciones, donde hay subculturas y surgen conflictos entre las áreas y/o grupos formales e informales. Y, tercero, fragmentación, en la que la relación con las manifestaciones culturales es ambigua, el consenso es transitorio y específico para cada problema; los términos claves para esta perspectiva son redes de ambigüedad, paradoja y contradicción.

Adicional a estos análisis, Peiró (1990) y Alcover (2008) en la definición de cultura organizacional incluyen sus contenidos, elementos y/o niveles de análisis. Y, nuevamente, de acuerdo con Peiró (1990), surgen tres niveles que sirven para intentar aclarar los contenidos culturales de las organizaciones: 1) nivel observable, en el que lo evidente corresponde a los productos/artefactos de la cultura: lenguajes, rituales, sanciones, normas, patrones de conducta, costumbres, clima, símbolos, artefactos, mitos, leyendas, espacio físico, vestido, dotación, entre otros. 2) Nivel apreciativo y valorativo: son los lineamientos de la organización como, por ejemplo, valores, filosofía, ideología, expectativas, actitudes, perspectivas, conocimientos, prioridades, significados, etc. 3) Nivel fundante: son los supuestos y creencias básicas, que no se hacen tan evidentes en las manifestaciones culturales, son elementos esenciales de la cultura entre sí.

Rousseau (1997), propone, también, una clasificación de los principales elementos de la cultura, que van desde los de menor accesibilidad y mayor subjetividad a los de mayor accesibilidad y mayor objetividad; estos son presunciones fundamentales, valores, normas de comportamiento, patrones de conducta y artefactos.

Cuando se revisa la literatura científica, se encuentran diversas discusiones sobre los diferentes abordajes para definir y describir la cultura en las organizaciones. Buscar un acuerdo en sus definiciones y conceptos resulta casi imposible; esto deja 
en evidencia la vulnerabilidad del tema frente a los que tratan de deslegitimar la investigación en este campo (Alcover, 2008).

Si esto ocurre con su definición, igual pasa con su medición. Frente a las mediciones de la cultura se encuentran las dimensiones que se han utilizado para abordar de forma empírica su estudio. Con base en los planteamientos de uno de los autores más representativos, Geert Hofstede (2001), una dimensión es un aspecto de una cultura que puede medirse con relación a otras culturas. Los intentos por definir dimensiones culturales provienen de estudios de antropólogos, sociólogos y psicólogos sociales; la que ha tenido mayor difusión y aceptación en las últimas tres décadas es la propuesta por Hofstede; es una investigación transcultural realizada entre organizaciones multinacionales pertenecientes a 50 países (Hofstede, 1980; 2001). Las dimensiones que incluyó Hofstede (1980), en el estudio fueron cuatro:

\begin{abstract}
1) distancia jerárquica o de poder, que mide la desigualdad social y relaciones con la autoridad; 2) colectivismo frente a individualismo, y mide relaciones entre individuo y grupo; 3) feminidad frente a masculinidad, mide implicaciones sociales de pertenecer a uno u otro género; 4) control de la incertidumbre, mide las formas de tratar la incertidumbre relacionadas con el control de la agresión y las expresiones de las emociones. (p. 48)
\end{abstract}

Unos años más tarde, Hofstede y Bond (1988) identificaron otra dimensión, orientación a largo plazo versus orientación a corto plazo, en la vida.

También se identificaron otros esfuerzos por utilizar dimensiones de la cultura nacional para la cultura organizacional, sin embargo, el mismo Hofstede (1991) señala que: "los resultados de las investigaciones realizadas sobre culturas nacionales han demostrado ser solo parcialmente útiles para comprender las culturas organizacionales" (pp. 54-55).

Posteriormente, en 1991, R. J. House concibió el programa de investigación Global Leadership and Organizational Behavior Effectiveness (Globe), el cual estudia las interrelaciones del liderazgo, la cultural societal y la cultura organizacional (House et al., 1999), con una muestra que ha llegado a más de 20.000 personas, más de 800 organizaciones y más de 60 culturas. La mayoría de las revisiones teóricas, epistemológicas y metodológicas alrededor de la cultura organizacional se dirigen indistintamente al proyecto Globe (Globe, 2016).

Se pueden buscar y reseñar otros trabajos sobre cultura organizacional, pero la relación que se pretende presentar y resaltar en este capítulo es el liderazgo y la cultura organizacional, y ambos conceptos están asociados a un enfoque cultural, en el que el 
liderazgo es el motor. De acuerdo con Alcover (2008), el líder es responsable de crear y transmitir los elementos de la cultura organizacional como la visión, la misión, los valores, las normas, etc., así como las prácticas de los procesos que se pretende establecer. Por ejemplo, el profesor Molero (2002) afirma que las relaciones entre cultura y liderazgo pueden verse desde tres perspectivas y niveles: culturas transnacionales, nacionales y subnacionales.

Tabla 5. Perspectivas y niveles de la cultura según Molero (2002)

\begin{tabular}{cc}
\hline Tipo de cultura & Características \\
\hline Culturas transnacionales & Compartidas por los miembros de diferentes países \\
Culturas nacionales & Las que comparten los miembros de un mismo país \\
Culturas subnacionales & Las que son compartidas por los miembros de un departamento, \\
& área o unidad dentro de una organización \\
\hline
\end{tabular}

Fuente: Aguilar (2017).

Lo que se ha logrado identificar es que los estudios y las investigaciones entre liderazgo y cultura se llevan a cabo en dos vías, una, cuando la cultura determina el tipo de liderazgo que surge, y dos, cuando el liderazgo determina la cultura. En la primera, Bass (1990), Triandis (1995) y Smith y Bond (1998), evidencian que esta perspectiva es en la que se han realizado la mayor cantidad de trabajos en los últimos años y, esto ha facilitado hacer comparaciones transculturales y/o transorganizacionales.

Para la segunda perspectiva, en la que el liderazgo determina la cultura, Schein (1985), afirma que: "cultura y liderazgo son dos caras de la misma moneda" (p. 2), lo más importante que hacen los líderes es crear y manejar las culturas. Así, cuando se revisa esta relación, resulta muy relevante tener en cuenta el tipo de cultura sobre la que se hace el análisis, y si se considera variable independiente o dependiente con respecto al liderazgo.

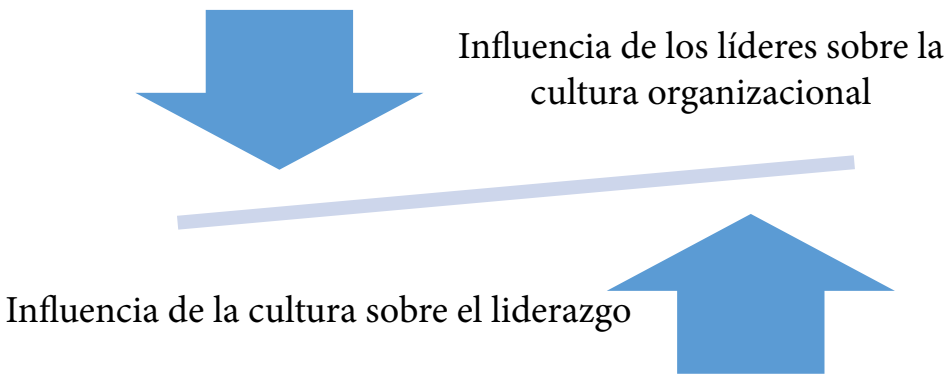

Figura 1. Relación entre cultura organizacional y liderazgo

Fuente: Aguilar (2017) 


\section{Conclusión}

Definitivamente, lo que resulta relevante es que la cultura influye en el liderazgo y el liderazgo influye en la cultura, esto no se puede negar. Sin embargo, todavía queda mucho por estudiar, investigar, explicar y entender. Se requieren más trabajos que analicen estas influencias y la forma en que las personas ejercen el liderazgo; investigaciones que expliquen cómo la cultura influye sobre el liderazgo en el contexto organizacional. Seguir explorando y estudiando estos temas ha sido una realidad, pero deben enriquecerse las investigaciones futuras desde una perspectiva del rol cambiante del líder de acuerdo con cada organización y a lo largo del tiempo, así como desarrollar metodologías más complejas e integrar otras variables psicológicas que son determinantes en los dos temas, y que han sido revisadas.

\section{Referencias}

Aditya, R. N., House, R. J. y Kerr, S. (2000). Theory and practice of leadership: Into the new millennium. En L. Cooper y E. Locke (eds.), Industrial and Organizational Psychology. Linking theory and practice (pp. 130-165). Blackwell Pub.

Adler. N. J., Doktor, R. y Redding, S. G. (1986). From the Atlantic to the Pacific century. Cross-cultural management reviewed. Journal of Management, 12, 295-318.

Aguilar Bustamante, M. C. (2017). Estudio comparativo del estilo de liderazgo y la cultura organizacional en asociaciones gremiales y directores de programas en psicología en Latinoamerica y España, con base en el proyecto Globe [tesis de doctorado Universidad de La Laguna].

Aguilar Bustamante, M. C. y Correa-Chica, A. (2017). Análisis de las variables asociadas al estudio del liderazgo: una revisión sistemática de la literatura. Universitas Psychologica, 16 (1), 1-13. http://dx.doi.org/10.11144/Javeriana.upsy16-1.avae

Allaire, Y. y Firsirotu, M. (1984). Theories of organizational culture. Organization Studies, 5(3), 193-226. doi: 10.1177/017084068400500301

Alcover, C. M. (2008). Las organizaciones en las sociedades actuales. En F. Gil y C. M. Alcover (coords.), Introducción a la psicología de las organizaciones (pp. 85-107). Alianza.

Anastácio-Barata, M. y Martí-Ripoll, M. (2015). El liderazgo en una perspectiva internacional: Un estudio comparativo entre líderes brasileños y españoles de acuerdo con el proyecto Globe. Revista de Psicología, 2(1), 35-49. http://periodicos.ufc.br/index.php/ psicologiaufc/article/view/1500

Antonakis, J., Cianciolo, A. T. y Sternberg, R. J. (2004). Leadership: Past, present, and future. En J. Antonakis, A. T. Cianciolo y R. J. Sternberg (eds.), The nature of leadership (pp. 3-15). Sage.

Bass, B. M. (1985). Leadership and performance beyond expectations. Free Press. 
Bass, B. M. (1990). Bass \& Stogdill's handbook of leadership: Theory, research, and managerial applications (3. ${ }^{\mathrm{a}}$ ed.). Free Press.

Bass, B. M. (1999). Two decades of research and development in transformational leadership. European Journal of Work and Organizational Psychology, 8(1), 9-32. http://dx.doi. org/10.1080/135943299398410

Bass, B. M. y Avolio, B. J. (1994). Improving organizational effectiveness through transformational leadership. Sage Publications.

Bligh, M.C. y Meindl, J.R. (2005). The cultural ecology of leadership: An analysis of popular leadership books. En D. M. Messick y R. M. Kramer (eds.), The psychology of leadership: New perspectives and research (pp. 11-51). Sage.

Bryman, A. (1996). Leaderships in organizations. En S. R. Clegg, C. Hardy y W. R. Nord (ed.). Handbook of Organizational Studies (pp. 276-292). Sage.

Child, J. D. y Tayeb, M. (1983). Theoretical perspectives in cross-national research. International Studies of Management and Organization, 23(4), 32-70.

Cunha, R. C., y Cooper, C. L. (1998). Privatization and the human factor. Journal of Applied Management Studies, 7, 201-210

Contreras, F., Barbosa, D., Juárez, F y Uribe, A. (2010). Efectos del liderazgo y del clima organizacional sobre el riesgo psicosocial, como criterio de responsabilidad social, en empresas colombianas del sector salud. Revista Argentina de Clínica Psicológica, 19(2), 173-182. http://www.redalyc.org/articulo.oa?id=281921801007

Contreras, F., Barbosa, D. y Piñeros, R. (2016). Liderazgo: antecedentes, tendencias y perspectivas de desarrollo. Editorial Universidad del Rosario.

Contreras, F. (2008). Liderazgo: perspectivas de desarrollo e investigación. International Journal of Psychological Research, 1(2) 64-72. http://www.redalyc.org/articulo. oa?id=299023508008 Contreras

Davenport, T. H. (2000). El trabajo del conocimiento y el futuro de la dirección. En W. Bennis, G. M. Spreitzer y T. G. Cummings (comps.), El futuro del liderazgo. (pp. 53-71). Deusto.

Deal, T. E. y Kennedy, A. A. (1982). Corporate cultures: The rites and rituals of corporate life. Penguin Books.

Delgado, L. y Castañeda, D. I. (2011). Relación entre capital psicológico y la conducta de compartir conocimiento en el contexto del aprendizaje organizacional. Acta Colombiana de Psicología, 14, 61-70. http://www.redalyc.org/articulo.oa?id=79822602006

Den Hartog, D. N. y Koopman, P. L. (2001). Leadership in organizations. En N. Anderson, D. S. Ones, H. Kepir-Sinangil y C. Viswesvaran, C. (eds.), International handbook of industrial, work and organizational psychology (vol. 2). Sage.

Denison, D. (1996). What is the difference between organizational culture and organizational climate? A native's point of view on a decade of paradigm wars. Academy of Management Review, 21, 619-654. doi: 10.5465/AMR.1996.9702100310 
Denison, D. y Mishra, A. (1995). Toward a theory of organizational culture and effectiveness. Organizational Science, 6(2), 204-223. http://www.trustiseverything.com/wp-content/ uploads/2012/07/denison-mishra-toward-a-theory-of-org-culture-and-effect-orgsci-1995.pdf

Dorfman, P. y Hanges, P. (2004). The identification of culturally endorsed leadership profiles. En R. J. House, P. J. Hanges, M. Javadin, P. W. Dorfman y V. Gupta. Culture, leadership and organizations: The Globe Study of 62 Societies (pp. 669-719). Sage.

Fiedler, F. E. (1967). A theory of leadership effectiveness. McGraw-Hill.

Fiedler, F. y García, J. (1987). New approaches to leadership, cognitive resources and organizational performance. John Wiley and Sons.

García-Rubiano, M., Aguilar Bustamante, M.C., Payan Wilson, E. y Forero, D. (2016). Investigación bibliométrica de los trabajos de grado sobre liderazgo en las facultades de psicología de la ciudad de Bogotá (Colombia). Universidad y Empresa, 17(28), 147-172. DOI: http://dx.doi.org/10.12804/rev.univ.empresa.28.2015.07

Gil, F. (2008). Dirección y liderazgo. En F. Gil y C. M. Alcover, Introducción a la psicología de las organizaciones (pp. 285-318). Alianza.

Gil, F. y Alcover, C. M. (2008). Introducción a la psicología de las organizaciones. Alianza.

Gil Rico, A. (2000). El programa de investigación Globe. Una estrecha relación entre cultura y liderazgo. Revista Reflexiones, 14, 30-92. http://uexternado2.metarevistas.org/index. php/sotavento/article/viewFile/1627/1466

Gil, F. y García Sáiz, M. (1993). Habilidades de dirección en las organizaciones. Eudema.

Gil, F. y Martí, M. (2011). Cultura y liderazgo. El proyecto Globe. En F. Molero y J. F. Morales (coords.), Liderazgo: hecho y ficción (pp. 197-220). Alianza.

Gil, F., Alcover, C., Rico, R. y Sánchez-Manzanares, M. (2011). Nuevas formas de liderazgo en equipos de trabajo. Papeles del Psicólogo, 32, 38-47. http://www.papelesdelpsicologo. es/pdf/1917.pdf

Globe (2016). Culture groups. Global leadership \& organizational behavior effectiveness. Autor. http://GLOBEproject.com/results/clusters/anglo?menu=list

Glynn, M. A. y De Jordy, R. (2010). Leadership through an organizational behavior lens: A look at the last half century of research. En N. Noria y R. Khurana (eds.), Handbook of Leadership Theory and Practice (pp. 119-157). Harvard Business Press.

Hanges, P. J. y Dickson, M. W. (2004). The development and validation of the Globe Culture and Leadership Scale. En M. Javidan, P. W. Dorfman y V. Gupta, Culture, leadership, and organizations. The Globe study of 62 societies (pp. 122-145). Sage.

Harrison, R. (1972). Understanding your organisation's character. Harvard Business Review, May-June, 119-128.

Hofstede, G. (1980). Culture's consequences: International differences in work related values. Sage. 
Hofstede, G. (2001). Culture's consequences: Comparing values, behaviors, institutions, and organizations across nations. Sage Publications.

Hofstede, G. y Bond, M. (1988). The Confucius connection: From cultural roots to economic growth. Organizational Dynamics, 16(4), 4-21.

Hosking, D. M. (1988). Organizing, leadership and skilful processes. Journal of Management Studies, 25, 147-166. doi: 10.1111/j.1467-6486.1988.tb00029.x

House, R. J. y Aditya, R. N. (1997). The social scientific study of leadership: Quo vadis?, Journal of Management, 23, 409-473. doi: 10.1177/014920639702300306

House R. J., Hanges P. J., Ruiz-Quintanilla, S. A., Dorfman, P. W., Javidan, M., Dickson, M., Gupta, V. y 170 co-authors. (1999). Cultural influences on leadership and organizations: Project Globe. En W. Mobley (ed.), Advances in global leadership, vol. 1. JAI Press.

House, R. J., Javidan M. y Dorfman, P. (2001). Project Globe: An Introduction. A p p li ed Psychology: An International Review, 50 (4), 489-505. doi: 10.1111/1464-0597.00070

House, R.J. y Javidan, M., (2002). Leadership and cultures around the world: findings from Globe. An introduction to the special issue. Journal of World Business, 37, 1-2. http:// ac.elscdn.com.ez.urosario.edu.co/S1090951601000682/1-s2.0-S1090951601000682main.pdf?_tid=1a35083a-24db-11e5-8714-00000aacb361\&acdnat $=1436296099$ _ df44908ac11cfb0b2a8de22809b34773

House, R. J., Wright, N. S., y Aditya, R. N. (1997). Cross-cultural research on Organizational leadership: A critical analysis and a proposed theory. En P. C. Earley y M. Erez (eds.), New perspectives in international industrial organizational psychology (pp. 535-625). New Lexington.

Katz, D. y Kahn, R. (1978). The social psychology of organizations. Wiley.

Katzenbach, J. R. y Smith, D. K. (1993). Sabiduría de los equipos. El desarrollo de la organización de alto rendimiento. Díaz de Santos.

Kerr, S. y Jermier, J. M. (1978). Substitutes for leadership: Their meaning and measurement. Organizational Behavior and Human Performance, 22, 375-403.

Kouzes, J. M., y Posner, B. Z. (1995). The leadership challenge: How to keep getting extraordinary things done in organizations. Jossey-Bass.

Kouzes, J. M., y Posner, B. J. (2002). Leadership challenge (3. a ed.). Jossey-Bass.

Levitt, T. (1983). The globalization of markets. Harvard Business Review, 83(3), 92-102.

Lord, R. y Maher, K. J. (1991). Leadership and information processing: Linking perceptions and performance. Unwin-Everyman.

Martín, J. (2002). Organizational culture, mapping the terrain. Sage.

Martínez Avella, M. (2010). Relaciones entre cultura y desempeño organizacional en una muestra de empresas colombianas: reflexiones sobre la utilización del modelo de Denison. Cuadernos de Administración, 23(40). http://revistas.javeriana.edu.co/index. php/cuadernos_admon/article/view/3625

Manz, C. C. y Sims, H. P. (1989). Superliderazgo. Paidós. 
Molero, F. (2002). Cultura y liderazgo. Una relación multifacética. Boletín de Psicología, 76, 53-75. http://www.uv.es/seoane/boletin/previos/N76-4.pdf

Molero, F. (2011). La investigación del liderazgo en psicología. En F. Molero y J. F. Morales (coords.), Liderazgo: hecho y ficción. (pp. 21-45). Alianza.

Molero, F. y Morales, J. F. (2011). Liderazgo: hecho y ficción. Alianza.

Moss, R. (2010). Leadership in a globalizing world. En N. Nohria y R. Khurana (eds.), Handbook of Leadership Theory and Practice (pp. 3-25). Harvard Business.

Nader, M. y Solano, A. (2009). Relación entre los estilos liderazgo, valores y cultura organizacional: un estudio con líderes civiles y militares. Anuario de Psicología, 40(2), 237-254. http://www.redalyc.org/pdf/970/97017660007.pdf

Ogliastri, E. (1999). Culture and organizational leadership in Colombia. En R. J. House y J. Chokkar, Cultures of the world, a Globe anthology of in-depth descriptions of the cultures of 14 countries. The Leadership Quarterly, 10(2), 219-256. doi https://doi.org/10.1016/ S1048-9843(99)00018-1

O’Reilly, C. A., Chatman, J., y Caldwell, D. F. (1991). People and organizational culture: A profile comparison approach to assessing person-organization fit. Academy of Management Journal, 34(3), 487-516. http://dx.doi.org/10.2307/256404

Peiró, J. M. (1990). Organizaciones: nuevas perspectivas psicosociológicas. PPU.

Peiró, J. M. (1995). Psicología de la organización (tomo II). UNED.

Rousseau, D. (1997). Organizational behavior in the new organizational era. Annual Review of Psychology, 48, 515-546. doi: 10.1146/annurev.psych.48.1.515

Sabucedo, J. M. y Morales, J. F. (2015). Psicología social. Editorial Médica Panamericana.

Schein, E. H. (1985). La cultura empresarial y el liderazgo. Plaza y Janés.

Schein, E. H. (1992). Organizational culture and leadership: A dynamic view (2. ${ }^{\mathrm{a}}$ ed.). Jossey-Bass.

Schilling, J. (2007). Leaders' romantic conceptions of the consequences of leadership. Applied Psychology, 56(4), 602-623. doi: http://dx.doi.org/10.1111/j.1464-0597.2007.00306.x

Schneider, B. (2000). The psychological life of organizations. En N. M. Askanasy, C. P. M. Wilderon y M. F. Peterson (eds.), Handbook of Organizational Culture and Climate (pp. xvii-xxi). Sage.

Shamir, B. y Howell, J. M. (1999). Organizational and contextual influences on the emergence and effectiveness of charismatic leadership. The Leadership Quarterly, 10(2), 257-283. http://dx.doi.org/10.1016/S1048-9843(99)00014-4

Shamir, B., House, R. J. y Arthur, M. B. (1993). Motivational effects of transformational leadership: A self-concept based theory. Organization Science, 4(4), 577-594.

Shiraev, E. B. y Levy, D. A. (2010). Cross-cultural psychology: Critical thinking and application (4. ${ }^{\mathrm{a}}$ ed.). Pearson/Allyn Bacon. 
Stogdill, R. M. (1974). Handbook of leadership: A survey of theory and research. Free Press. http://psycnet.apa.org/psycinfo/1974-22876-000

Smircich, L. (1983). Concepts of culture and organizational analysis. Administrative Science Quarterly, 28, 339-358. doi: 10.2307/2392246

Smith, P. B. y Bond, M. H. (1998). Social psychology across cultures. Prentice-Hall.

Smith, P. B. y Peterson, M. F. (1988). Leadership, organizations and culture: An event management approach. Sage.

Triandis, H. C. (1995). Individualism and collectivism. Westview Press.

Triandis, H. C. (2004). Foreword. En R. J. House, P. J. Hanges, M. Javidan, P. W. Dorfman y V. Gupta (eds.), Leadership, culture and organizations: The Globe study of 62 societies (pp. $\mathrm{xv}$-xix). Sage.

Trice, H. y Beyer, J. (1993). The cultures of work organization. Prentice Hall.

Vroom, V. H. y Jago, A. G. (1978). On the validity of the Vroom-Yetton model. Journal of Applied Psychology, 63, 151-162.

Vroom, V. y Yetton, P. W. (1973). Leadership and decision-making. University of Pittsburgh Press.

Yukl, G. (2002). Leadership in organizations. Prentice Hall Inc.

Yukl, G. y Van Fleet, D. D. (1992). Theory and Research on Leadership in Organizations. En M. D. Dunnette (ed.), Handbook of Industrial and Organizational Psychology. John Wiley \& Son. 